\title{
ATIVIDADE FITOTÓXICA DE Guettarda uruguensis (RUBIACEAE) SOBRE A GERMINAÇÃO E CRESCIMENTO DE SEMENTES DE Lactuca sativa
}

\author{
PHYTOTOXIC ACTIVITY OF Guettarda uruguensis (RUBIACEAE) IN THE \\ GERMINATION AND GROWTH OF SEEDS FROM Lactuca sativa
}

\section{Ana Flávia Schvabe DUARTE ${ }^{1}$; Mariana Saragioto KRAUSE¹; Antonio Luiz da Costa

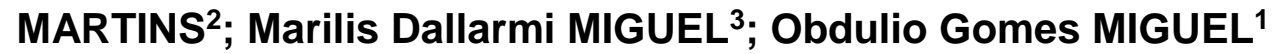

1 - Universidade Federal do Paraná, Departamento de Farmácia, Laboratório de Fitoquímica, Curitiba, PR, Brasil. 2 - Hospital Santa Casa de Misericórdia de Curitiba, Curitiba, PR, Brasil.

3 - Universidade Federal do Paraná, Departamento de Farmácia, Laboratório de Farmacotécnica, Curitiba, PR, Brasil

\section{RESUMO:}

O estudo avalia a atividade fitotóxica do extrato etanólico e respectivas frações orgânicas obtidas das cascas do caule da espécie Guettarda uruguensis Cham. \& Schltdl. (Rubiaceae) sobre a germinação e o crescimento de sementes de Lactuca sativa. Os parâmetros avaliados foram o índice de velocidade de germinação, crescimento da radícula e do hipocótilo. Os resultados obtidos no presente trabalho demonstram que Guettarda. uruguensis produz compostos químicos que atuam na estimulação e inibição da germinação das sementes, crescimento da radícula e inibição do hipocótilo da espécie Lactuca sativa.

Palavras-chave: Guettarda uruguensis, fitotoxicidade; Lactuca sativa.

\section{ABSTRACT:}

The study evaluates the phytotoxic activity of the ethanolic extract and its organic fractions obtained from the stem bark of the Guettarda uruguensis species Cham. \& Schltdl. (Rubiaceae) on germination and seed growth of Lactuca sativa. The evaluated parameters were the rate of germination velocity, radicle and hypocotyl growth. The results obtained in the present study demonstrate that Guettarda uruguensis produces chemical compounds that act in the stimulation and inhibition of the seeds germination, the radicle growth and inhibition of the hypocotyl of the Lactuca sativa species.

Keyworks: Guettarda uruguensis, phytotoxic; Lactuca sativa.

\section{INTRODUÇÃO}

As plantas sintetizam e degradam compostos orgânicos por via de reações anabólicas e catabólicas. Os compostos essências para a sobrevivência, tais como açucares, aminoácidos, ácidos graxos, nucleotídeos e polímeros derivados, constituem o metabolismo primário. De modo fundamental, os metabólitos primários estão associados à fotossíntese, respiração, crescimento e desenvolvimento da planta. Entretanto, o reino 
vegetal possui rotas biossintéticas secundárias, que ocorrem com elevado gasto de energético, associadas a produção dos metabólitos secundários. Em geral, estes compostos apresentam baixo peso molecular, ocorrem relativamente em baixas concentrações, são necessários para a sobrevivência, preservação e reprodução das espécies vegetais, representando uma expressão da individualidade (RAVEN et al., 2001; DEWICK, 2002; VON POSER \& MENTZ, 2004).

A princípio, a sobrevivência de uma espécie vegetal está assegurada pela garantia da reprodução e defesa de predadores, da radiação solar, entre outros. Entretanto, as interações planta-planta, fenômeno conhecido com o alelopatia, despenha um importante papel dentro desse contexto. Diante da riqueza da biodiversidade nacional, as plantas representam uma reserva de compostos químicos que podem apresentar potencial alelopatico, podendo servir como modelo na produção de novos herbicidas (MACIAS et al., 2000; VON POSER \& MENTZ, 2004; DUTRA et al., 2016).

Nesse sentido, a observação de efeitos fitotóxicos com enfoque alelopático possibilita a seleção de plantas que exerçam controle sobre espécies ou que atuem na composição de plantações visando equilíbrio biológico. A alelopatia tem atuação ecológica, especialmente em relação ao seu potencial herbicida, atuando como alternativa ao uso excessivo de agrotóxicos na agricultura, extremamente prejudiciais à saúde (SOUZA FILHO \& ALVES, 2002; MACIAS et al., 2000.)

Observada a importância da pesquisa de herbicidas naturais e ainda dentro desta perspectiva, este trabalho teve como objetivo verificar a atividade alelopática do extrato etanólico e frações de Guettarda uruguensis, na germinação e crescimento inicial de Lactuca sativa.

Guettarda uruguensis, conhecida popularmente como veludinho, é uma planta nativa do Brasil. É uma espécie ornamental que possui flores aromáticas produtoras de néctar, indicadas para a criação de abelhas sem ferrão e frutos comestíveis, sendo indicada para compor reflorestamentos heterogêneos de áreas degradadas. Com relação as atividades biológicas, o extrato etanólico obtido da casca do caule de G. uruguensis e frações orgânicas apresentam potencial antioxidante, antimicrobiano (DUARTE et al., 2014) e um perfil fitoquímico diversificado, indicando a presença de alcaloides, cumarinas taninos e heterosídeos saponínicos e cianogenéticos (DUARTE, 2012).

\section{MATERIAL E MÉTODOS}

\subsection{Material vegetal}

As cascas do caule de Guettarda uruguensis foram coletadas em Março de 2011 no Campus Jardim Botânico da Universidade Federal do Paraná, Brasil. O material vegetal 
foi identificado pela exsicata MBM 342335 do Herbário do Museu Botânico Municipal de Curitiba.

\subsection{Preparo do extrato etanólico e frações orgânicas}

O extrato etanólico foi obtido a partir de $1190 \mathrm{~g}$ de material vegetal, por meio de aparelho de Soxhlet (CARVALHO et al., 2009) utilizando como líquido extrator etanol $96^{\circ} \mathrm{GL}$. As frações orgânicas foram obtidas por particionamento do extrato etanólico, por meio de aparelho de Soxhlet (CARVALHO et al., 2009) utilizando solventes em escala de polaridade crescente. Foram obtidas as frações hexano, diclorometano, acetato de etila e hidroalcoólica remanescente.

\section{Avaliação da atividade fitotóxica}

A atividade alelopatica do extrato etanólico e frações obtidas da casca do caule de Guettarda uruguensis foi avaliada com relação a germinação das sementes e crescimento da radícula e hipocótilo da espécie Lactuca sativa L. cv Babá de verão, de acordo com DIAS et al., (2005). As amostras foram preparadas em diferentes concentrações (400, 200, 100 e $50 \mu \mathrm{g} / \mathrm{mL}$ ), utilizando metanol como solvente. Os experimentos foram realizados em câmera de germinação $\left(20^{\circ} \mathrm{C}\right.$; ausência de luz) utilizando caixas Gerbox® forradas com papel de filtro estéril umedecido com $1 \mathrm{~mL}$ da amostra, mantidas à $60^{\circ} \mathrm{C}$ por $24 \mathrm{~h}$ para evaporação do solvente com posterior adição de $3 \mathrm{~mL}$ de água destilada estéril e 20 sementes de Lactuca sativa. O índice de velocidade de germinação (IVG) foi calculado diariamente, durante sete dias, para cada repetição de cada tratamento (MAGUIRE, 1962). As sementes foram consideradas germinadas quando se tornaram visíveis a protrusão da radícula através do tegumento (DE FEO et al., 2002). A medida do crescimento da radícula e do hipocótilo foi realizada no sétimo dia do experimento. Os dados foram submetidos à análise de variância seguido do teste de Tukey utilizando o programa GrahPad Prism 5.Ink.

\section{RESULTADOS E DISCUSSÃO}

\subsection{Teste de Germinação}

No teste de germinação para Lactuca sativa L. foi determinado o IVG e os resultados obtidos para as amostras das cascas do caule de $G$. uruguensis podem ser visualizados no FIGURA 1 e TABELA 1. A análise dos resultados obtidos demonstra que 0 extrato etanólico e frações em todas as concentrações avaliadas diminuíram $(p<0,05)$ a 
velocidade de germinação das sementes de Lactuca sativa. Segundo Maguire (1962) o índice de velocidade de germinação (IVG) é um parâmetro para avaliação do vigor de sementes. Quando este é reduzido sugere-se uma perda progressiva da capacidade produtiva da semente e na uniformidade da germinação.

FIGURA 1 - ÍNDICE DE VELOCIDADE DE GERMINAÇÃO (IVG) DE Lactuca sativa L. NAS AMOSTRAS DE Guettarda uruguensis

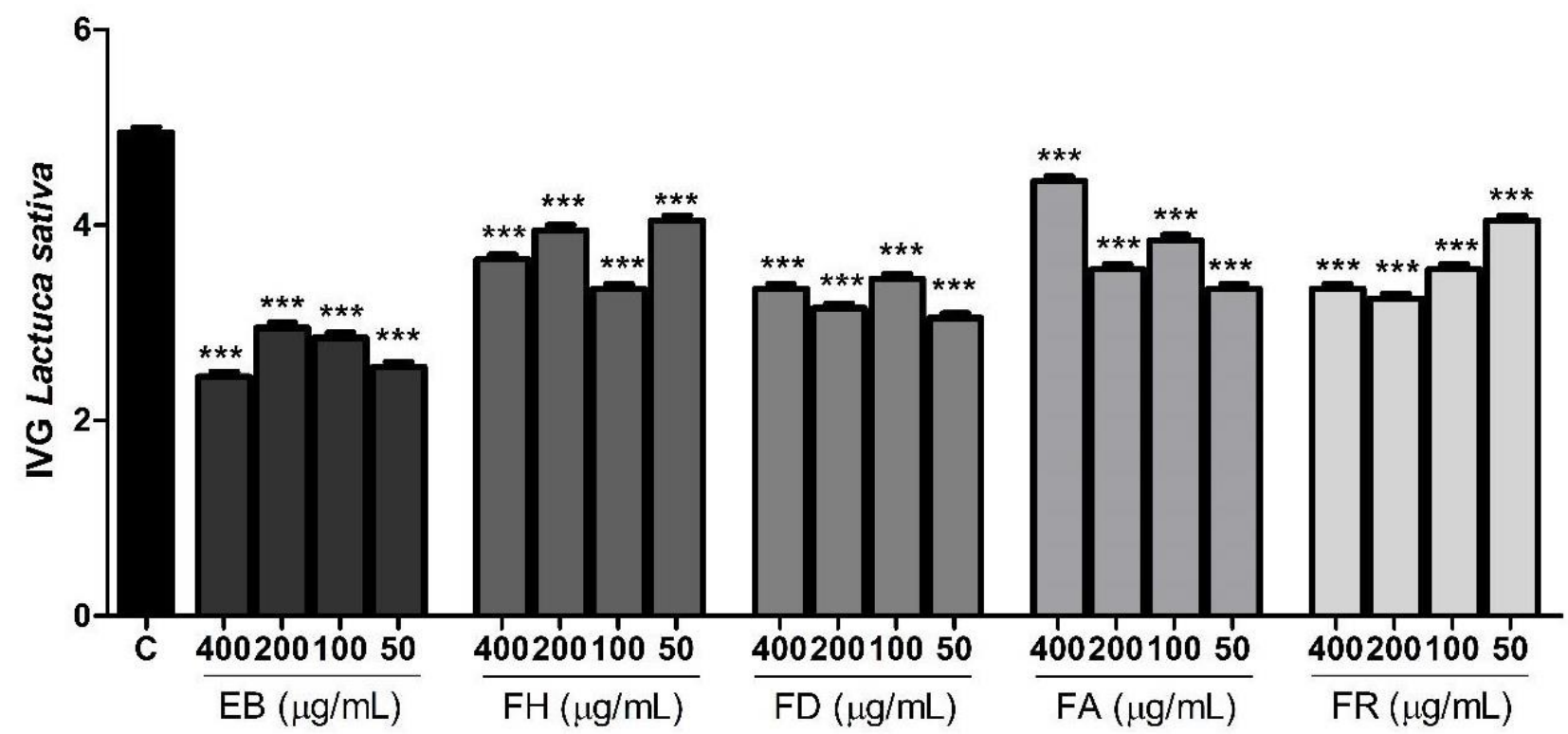

Legenda: EB: extrato etanólico; FH: fração hexano; FD: fração diclorometano; FA: fração acetato de etila; FRE: fração remanescente; C: controle água destilada. Os níveis de significância são indicados por ${ }^{* * *} p<0,001$ quando comparados com o controle (ANOVA de uma via seguida do teste de Tukey).

TABELA 1 - ÍNDICE DE VELOCIDADE DE GERMINAÇÃO (IVG) DE Lactuca sativa L. TRATADAS COM AMOSTRAS DE G. uruguensis

\begin{tabular}{l|c|c|c|c}
\hline \multirow{2}{*}{ EXTRATO/FRAÇÃO } & \multicolumn{4}{|c}{ Concentração $(\boldsymbol{\mu g} / \mathbf{m L})$} \\
\cline { 2 - 5 } & $\mathbf{5 0}$ & $\mathbf{1 0 0}$ & $\mathbf{2 0 0}$ & $\mathbf{4 0 0}$ \\
\hline Extrato etanólico & $2,625^{a}$ & $2,875^{a}$ & $3,000^{a}$ & $2,500^{a}$ \\
\hline Fração hexano & $4,125^{a}$ & $3,375^{a}$ & $4,000^{a}$ & $3,750^{a}$ \\
\hline Fração diclorometano & $3,125^{a}$ & $3,500^{a}$ & $3,250^{a}$ & $3,375^{a}$ \\
\hline Fração acetato de etila & $3,375^{a}$ & $3,875^{a}$ & $3,625^{a}$ & $4,500^{a}$ \\
\hline Fração remanescente & $4,083^{a}$ & $3,563^{a}$ & $3,333^{a}$ & $3,375^{a}$ \\
\hline
\end{tabular}

Legenda: Resultados em uma mesma linha quando seguidos pela(s) mesma(s) letra(s) não diferem estatisticamente $(p<0,05)$ entre si, pelo teste de Tukey. 


\subsection{Teste de crescimento}

No teste de crescimento de Lactuca sativa L. tratada com as amostras das cascas do caule de G. uruguensis, foram realizadas medições das radículas e hipocótilos e os valores em milímetros estão plotados nos GRÁFICOS 2 e 3. Em todos os ensaios realizados os controles expressaram resultados semelhantes $(p<0,05)$ descartando-se a influência sobre o crescimento do solvente metanol.

Os resultados demonstraram inibição $(p<0,05)$ do crescimento das radículas de Lactuca sativa L. expostas ao extrato etanólico na concentração de $50 \mu \mathrm{g} / \mathrm{mL}$, representando uma inibição de 58,3\% em relação ao grupo controle $(18,1 \pm 3,40 \mathrm{~mm})$. Promoveram estímulo $(\mathrm{p}<0,05)$ do crescimento da radícula a fração hexano $(100 \mu \mathrm{g} / \mathrm{mL})$ e frações diclorometano, acetato de etila e remanescente nas concentrações de 400, 200100 e $50 \mu \mathrm{g} / \mathrm{mL}$, conforme FIGURA 2 e TABELA 2.

FIGURA 2 - CRESCIMENTO DAS RADÍCULAS DE Lactuca sativa L. TRATADA COM EXTRATO ETANÓLICO E FRAÇÕES DE G. uruguensis.

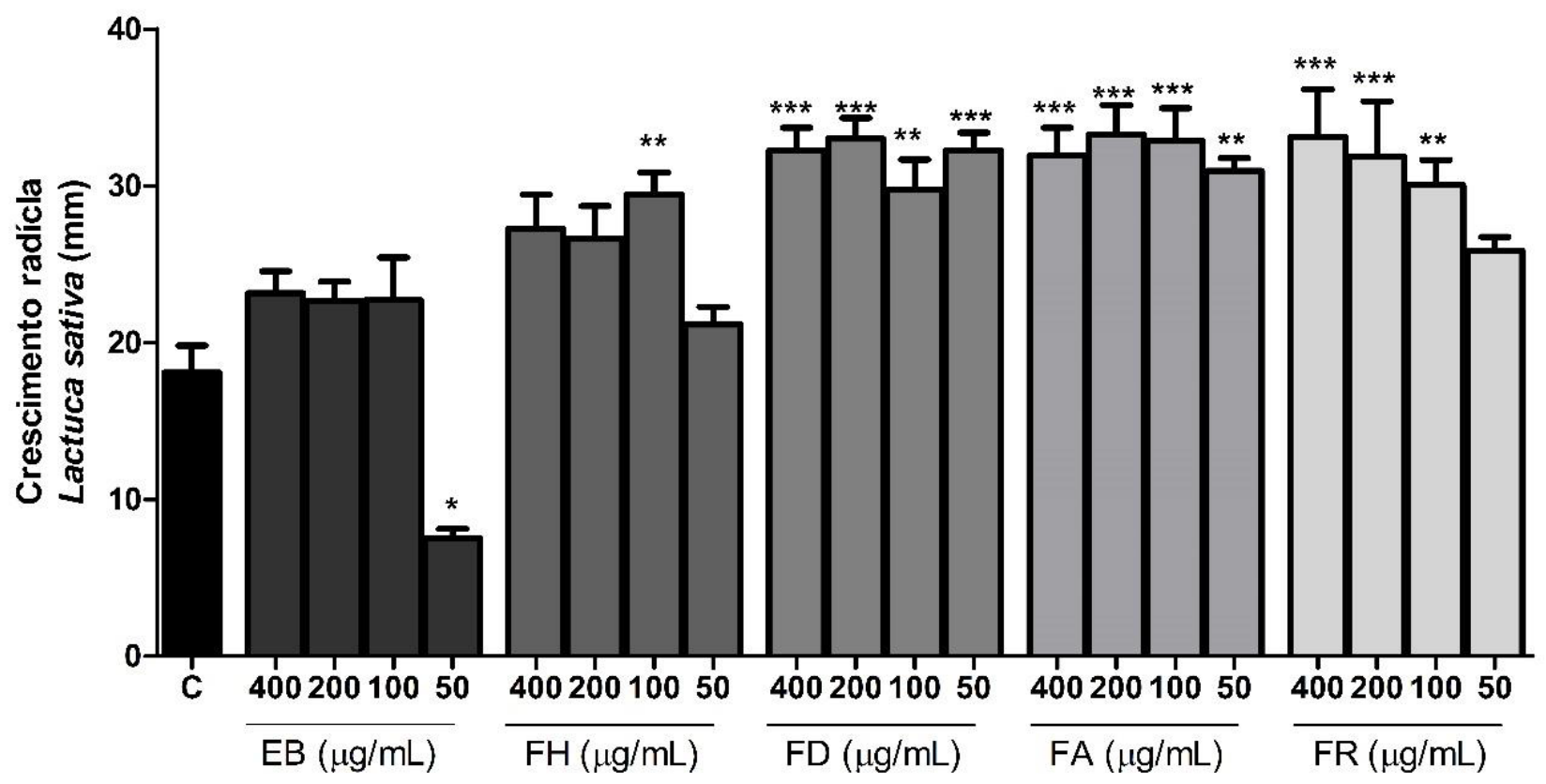

Legenda: EB: extrato etanólico; FH: fração hexano; FD: fração diclorometano; FA: fração acetato de etila; FRE: fração remanescente; C: controle água destilada. Os níveis de significância são indicados por ${ }^{*} p<0,05,{ }^{* *} p<0,01 e^{* \star *} p<0,001$ quando comparados com 0 controle (ANOVA de uma via seguida do teste de Tukey). 
TABELA 2 - CRESCIMENTO DAS RADÍCULAS DE Lactuca sativa L. TRATADA COM EXTRATO ETANÓLICO E FRAÇÕES DE G. uruguensis.

\begin{tabular}{l|c|c|c|c}
\hline \multirow{2}{*}{ EXTRATO/FRAÇÃo } & \multicolumn{4}{|c}{ Concentração $(\boldsymbol{\mu g} \mathbf{g} \mathbf{m L})$} \\
\cline { 2 - 5 } & $\mathbf{5 0}$ & $\mathbf{1 0 0}$ & $\mathbf{2 0 0}$ & $\mathbf{4 0 0}$ \\
\hline Extrato etanólico & $7,55 \pm 1,17^{b}$ & $22,70 \pm 5,48^{a}$ & $22,65 \pm 2,47^{a}$ & $23,15 \pm 2,82^{a}$ \\
\hline Fração hexano & $21,15 \pm 2,26^{b}$ & $29,45 \pm 2,78^{a}$ & $26,60 \pm 4,23^{a}$ & $27,25 \pm 4,36^{a}$ \\
\hline Fracão diclorometano & $32,252,27^{a}$ & $29,75 \pm 3,86^{a}$ & $33,00 \pm 2,66^{a}$ & $32,25 \pm 2,90^{a}$ \\
\hline Fração acetato de etila & $30,95 \pm 1,61^{a}$ & $32,85 \pm 4,22^{a}$ & $33,25 \pm 3,74^{a}$ & $31,95 \pm 3,49^{a}$ \\
\hline Fração remanescente & $25,85 \pm 1,81^{b}$ & $30,05 \pm 3,19^{a}$ & $31,85 \pm 7,08^{a}$ & $33,10 \pm 6,11^{a}$ \\
\hline
\end{tabular}

Legenda: Resultados em uma mesma linha quando seguidos pela(s) mesma(s) letra(s) não diferem estatisticamente $(p<0,05)$ entre si, pelo teste de Tukey.

Quanto ao crescimento dos hipocótilos, verifica-se inibição do crescimento $(p<0,05)$ em todas as concentrações das amostras avaliadas, conforme demonstrado no FIGURA 3, TABELA 3. A maior inibição do crescimento do hipocótilo ocorreu nas sementes de Lactuca sativa expostas ao extrato etanólico na concentração de $50 \mu \mathrm{g} / \mathrm{mL}(2,9 \pm 0,58 \mathrm{~mm})$, representando uma inibição de 90,1\% em relação ao grupo controle (29,27 £ 1,42 mm).

FIGURA 3 - CRESCIMENTO DO HIPOCÓTILO DE Lactuca sativa L. TRATADA COM EXTRATO ETANÓLICO E FRAÇÕES DE G. uruguensis.

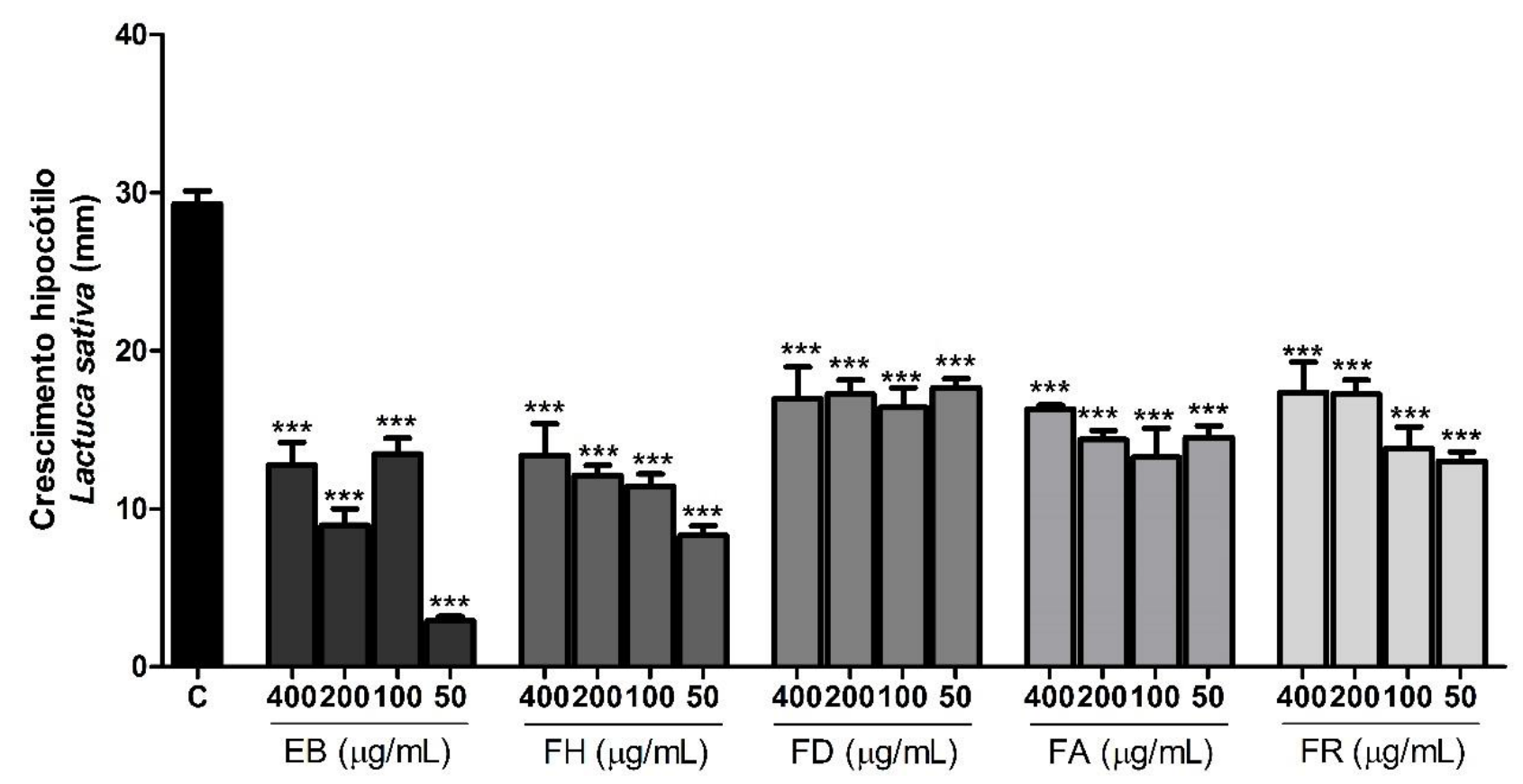

Legenda: EB: extrato etanólico; FH: fração hexano; FD: fração diclorometano; FA: fração acetato de etila; FRE: fração remanescente; C: controle água destilada. Os níveis de significância são indicados por ${ }^{* * *} p<0,001$ quando comparados com o controle (ANOVA de uma via seguida do teste de Tukey). 
TABELA 3 - CRESCIMENTO DO HIPOCÓTILO DE Lactuca sativa L. TRATADA COM EXTRATO ETANÓLICO E FRAÇÕES DE G. uruguensis.

\begin{tabular}{l|c|c|c|c}
\hline \multirow{2}{*}{ EXTRATO/FRAÇÃo } & \multicolumn{4}{|c}{ Concentração $(\boldsymbol{\mu g} \mathbf{g} \mathbf{m L})$} \\
\cline { 2 - 5 } & $\mathbf{5 0}$ & $\mathbf{1 0 0}$ & $\mathbf{2 0 0}$ & $\mathbf{4 0 0}$ \\
\hline Extrato etanólico & $2,85 \pm 0,58^{c}$ & $13,45 \pm 1,99^{a}$ & $8,95 \pm 2,09^{b}$ & $12,75 \pm 2,89^{a}$ \\
\hline Fração hexano & $8,30 \pm 1,21^{b}$ & $11,40 \pm 1,56^{a}$ & $12,10 \pm 1,27^{a}$ & $13,35 \pm 4,03^{a}$ \\
\hline Fração diclorometano & $17,65 \pm 1,11^{a}$ & $16,40 \pm 2,49^{a}$ & $17,25 \pm 1,74^{a}$ & $16,95 \pm 3,99^{a}$ \\
\hline Fração acetato de etila & $14,50 \pm 1,45^{a}$ & $13,25 \pm 3,65^{a}$ & $14,40 \pm 1,13^{a}$ & $16,30 \pm 0,50^{a}$ \\
\hline Fração remanescente & $13,00 \pm 1,17^{b}$ & $13,80 \pm 2,73^{b}$ & $17,25 \pm 1,74^{a}$ & $17,35 \pm 3,83^{a}$ \\
\hline
\end{tabular}

Legenda: Resultados em uma mesma linha quando seguidos pela(s) mesma(s) letra(s) não diferem estatisticamente $(p<0,05)$ entre si, pelo teste de Tukey

As sementes de Lactuca sativa expostas ao extrato e frações de G. uruguensis demonstraram influência significativa no crescimento, verificou-se que com exceção do extrato etanólico na concentração de $50 \mu \mathrm{g} / \mathrm{mL}$, a fração hexano $(100 \mu \mathrm{g} / \mathrm{mL})$ e frações diclorometano, acetato de etila e remanescente, em todos os níveis de concentração avaliados, atuaram positivamente no crescimento radicular, entretanto inibiram o crescimento do hipocótilo. Este desequilíbrio entre o crescimento da radícula e do hipocótilo pode impossibilitar o desenvolvimento, gerando plântulas anormais (FERREIRA; AQUILA, 2000).

Segundo Borella \& Pastorini (2009), a raiz é o órgão vegetal que apresentam maior sensibilidade à ação de aleloquímicos quando comparada com o hipocótilo da plântula, pelo fato de estarem em contato direto com a composto em teste. Entretanto, no presente estudo observou-se que somente a dose mais baixa utilizada $(50 \mu \mathrm{g} / \mathrm{mL})$ do extrato etanólico produziu uma influência negativa sob o crescimento da radícula, enquanto que as demais amostras demonstraram influência positiva sobre o mesmo parâmetro. Segundo Hoffmann et al., (2007), o alongamento da parte aérea e raiz é influenciado por processos de divisão celular, formação do câmbio e vasos xilemáticos, sendo essas estruturas dependentes da partição de nutrientes pela plântula. Ferreira e Áquila (2000) e Ferreira (2004), sugerem que os efeitos alelopáticos sob o crescimento estão relacionados a alterações de membranas, síntese proteica, respiração, conformação de moléculas, entre outros. Portanto, sugerimos que o extrato e frações de G. uruguensis atuem de maneira direta ou indireta em algumas destas estruturas.

Visto a importância da busca por alternativas menos tóxicas ao meio ambiente, os resultados obtidos estimulam a continuidade deste trabalho para avaliar o extrato etanólico e compostos isolados, quanto aos seus mecanismos de ação e potencial alelopático sobre outras espécies vegetais. 


\section{REFERÊNCIAS}

BORELLA, J.; PASTORINI, L.H. Influência alelopática de Phytolacca dioica L. na germinação e crescimento inicial de tomate e picão-preto. Revista Biotemas, v.22, n.3, p.6775, 2009.

CARVALHO, J.L.S.; CUNICO, M.M.; DIAS, J.F.G.; MIGUEL, M.D.; MIGUEL, O. G. Termstability of extractive processes from Nasturtium officinale R. Br., brassicaceae for Soxhlet modified system. Quim. Nova, v.32, n.4, p.1031-1035, 2009.

DE FEO, V.; DE SIMONE, F.; SENATORE F. Pottential allelochemicals from the essential oil of Ruta graveolens. Phytochemistry, v.61, n.5, p.573-578, 2002.

DEWICK, P.M. Medicinal Natural Products. 2. ed. John Wiley \& Sons, Ltd. UK, 2002.

DIAS, J.F.G. Estudo alelopático aplicado de Aster lanceolatus, Willd. Curitiba, 2005. Dissertação [Mestrado em Ciências Farmacêuticas] - Setor de Ciências da Saúde, Universidade Federal do Paraná.

DUARTE, A.F.S. Estudo fitoquímico, toxicidade e atividades biológicas (antioxidante, antimicrobiana e alelopática) de cascas do caule de Guettarda uruguensis Cham. \& Schltdl., Rubiaceae. 118f. Curitiba, 2012. Dissertação [Mestrado em Ciências Farmacêuticas] Setor de Ciências da Saúde, Universidade Federal do Paraná.

DUARTE, A.F.S.; HIROTA, B.C.K.; OLIVEIRA, V.B.; CAMPOS, R.; MURAKAMI, F.S.; MIGUEL, M.D.; MIGUEL, O.G. Avaliação da atividade antioxidante e antimicrobiana do extrato etanólico bruto e frações orgânicas obtidas a partir da casca do caule da espécie Guettarda uruguensis Cham. \& Schltdl. (Rubiaceae). Revista de Ciências Farmacêuticas Básica e Aplicada, v. 35, n. 4, p. 607-614, 2014.

DUTRA, R.C.; CAMPOS, M.M.; SANTOS, A.R.S.; CALIXTO, J.B. Medicinal plants in Brazil: Pharmacological studies, drug discovery, challenges and perspectives. Pharmacological Research, v.112, p.4-29, 2016. 
FERREIRA, A.G., AQUILA, M. E. Alelopatia: uma área emergente da ecofisiologia. Revista Brasileira de Fisiologia Vegetal, v.12, p.175-204, 2000.

FERREIRA, I.C.P.; LONARDONI, M.V.C.; MACHADO, G.M.C.; LEON, L. L.; GOBBI FILHO, L.; PINTO, L.H.B.; OLIVEIRA, A. J.B. Anti-leishmanial activity of alkaloidal extract from Aspidosperma ramiflorum. Memórias do Instituto Oswaldo Cruz, v. 99, n.3, p.325-327, 2004.

HOFFMANN, C.E.F., NEVES, L.A.S.; BASTOS, C.F.; WALLAU, G.L. Atividade alelopática de Nerium oleander L. e Dieffenbachia picta schott em sementes de Lactuca sativa L. e Bidens pilosa L. Revista de Ciências Agroveterinárias, v.6, n. 1, p.11-21, 2007.

MACIAS, F.A.; CASTELLANO, D.; MOLINILLO, J.M.G. Search for a standard phytotoxic bioassay for allelochemicals. Selection of standard target species. J Agric Food Chem., v.48, n.6, p.2512-21, 2000.

MAGUIRE, J.D. Speed of germination - aid in selection and evaluation for seedling emergence and vigor. Crop Science, v.2, n.2, p.176-177, 1962.

RAVEN, P.H.; EVERT, R.F.; EICHHORN, S.E. Biologia Vegetal. 6. ed. Rio de Janeiro, RJ: Guanabara Koogan, 2001.

SOUZA FILHO, A.P.S., ALVES, S.M. 2002. Alelopatia: princípios básicos e aspectos gerais. Belém: Embrapa Amazônia Oriental, 206 p.

VON POSER, G.L.; MENTZ, L.A. Diversidade Biológica e Sistemas de Classificação. In: Farmacognosia: da planta ao medicamento. SIMÕES, Cláudia Maria Oliveira; et al. Editora da UFRGS/Editora da UFSC: Porto Alegre/Florianópolis, 5. ed., p. 75-89, 2004. 